\title{
Cervical Cord Neurodegeneration in Traumatic and Non-Traumatic Spinal Cord Injury
}

\author{
Maryam Seif, ${ }^{1}$ Gergely David, ${ }^{1}$ Eveline Huber, ${ }^{1}$ Kevin Vallotton, ${ }^{1}$ Armin Curt, ${ }^{1}$ and Patrick Freund ${ }^{1-4}$
}

\begin{abstract}
This study aimed to compare macrostructural and microstructural neurodegenerative changes remote from a cervical spinal cord injury in traumatic spinal cord injury (tSCI) and degenerative cervical myelopathy (DCM) patients using quantitative magnetic resonance imaging (MRI). Twenty-nine tSCI patients, 20 mild/moderate DCM patients, and 22 healthy controls underwent a high-resolution MRI protocol at the cervical cord (C2/C3). High-resolution T2*-weighted and diffusion-weighted scans provided data to calculate tissue-specific cross-sectional areas of the spinal cord and tractspecific diffusion indices of cord white matter, respectively. Regression analysis determined associations between neurodegeneration and clinical impairment. tSCI patients showed more impairment in upper limb strength and manual dexterity when compared with DCM patients. While macrostructural MRI measures revealed a similar extent of remote cord atrophy at cervical level, microstructural measures (diffusion indices) were able to distinguish more pronounced tract-specific neurodegeneration in tSCI patients when compared with DCM patients. Tract-specific neurodegeneration was associated with upper limb impairment. Despite clinical differences between severely impaired tSCI compared with mildly affected DCM patient, extensive cord atrophy is present remotely from the focal spinal cord injury. Diffusion indices revealed greater tract-specific alterations in tSCI patients. Therefore, diffusion indices are more sensitive than macrostructural MRI measures as these are able to distinguish between traumatic and non-traumatic spinal cord injury. Neuroimaging biomarkers of cervical cord integrity hold potential as predictors of recovery and might be suitable biomarkers for interventional trials both in traumatic and non-traumatic SCI.
\end{abstract}

Keywords: biomarker; DCM; DTI; quantitative MRI; traumatic SCI

\section{Introduction}

$\mathbf{T}$ RAUMATIC SPINAL CORD INJURY (TSCI) and non-traumatic degenerative cervical myelopathy (DCM) are conditions that arise from focal cervical damage. ${ }^{1,2}$ The most obvious difference between a traumatic and non-traumatic cervical myelopathy lies in the time profile of neural changes (acute onset in tSCI vs. slowly developing symptoms in DCM). ${ }^{3-6}$ Due to progressive impairment of gait and the increasing risk of falls, DCM patients can develop a central cord syndrome, which per definition is a tSCI. ${ }^{7}$ Experimental evidence suggests that tSCI and DCM share several aspects of myelopathy with a combination of alpha-motoneuron damage (lesion of the central gray), ${ }^{8}$ demyelination, ${ }^{9-12}$ and axonal damage of long projecting spinal nerve fiber tracts (white matter damage), ${ }^{13,14}$ as well as edema and ischemic changes. ${ }^{11,15}$ Both etiologies present with varying degrees of upper limb im- pairment $^{16-18}$ that can be assessed by comprehensive clinical protocols sensitive to sensorimotor functions (e.g., Graded Redefined Assessment of Strength, Sensibility, and Prehension [GRASSP]). ${ }^{19,20}$ Although such advanced clinical assessment allows quantifying the degree of impairment, it cannot disclose the underlying pathophysiology that occurs at the microstructural level.

Quantitative magnetic resonance imaging (qMRI) shows potential to detect such specific (micro-) structural changes in the spinal cord, both in tSCI ${ }^{21-24}$ and in DCM patients. ${ }^{25-27}$ To compare the magnitude of injury-induced neurodegenerative changes in both etiologies, we applied high-resolution T2*-weighted MRI and diffusion tensor imaging (DTI) above the injury level. We hypothesized that tSCI patients should show a more pronounced pattern of neurodegenerative changes compared with DCM patients, in which the disease slowly develops over time.

\footnotetext{
${ }^{1}$ Spinal Cord Injury Center, Balgrist University Hospital, ${ }^{4}$ Department of Neurology,University Hospital Zurich, University of Zurich, Zurich, Switzerland.

${ }^{2}$ Department of Neurophysics, Max Planck Institute for Human Cognitive and Brain Sciences, Leipzig, Germany.

${ }^{3}$ Wellcome Trust Centre for Neuroimaging, UCL Institute of Neurology, London, United Kingdom.

(C) Maryam Seif et al., 2019; Published by Mary Ann Liebert, Inc. This Open Access article is distributed under the terms of the Creative Commons License (http://creativecommons.org/licenses/by/4.0), which permits unrestricted use, distribution, and reproduction in any medium, provided the original work is properly credited.
} 


\section{Methods}

\section{Participants and study design}

Patients with tSCI at cervical level $(n=29$; American Spinal Injury Association Impairment Scale [AIS] A-D; mean age $47.4 \pm$ standard deviation [SD] 19.8 years, five female) and patients with mild and moderate DCM $(n=20$, AIS D; mean age $52.0 \pm 14.5$ years; six female) were enrolled in this study at the University Hospital Balgrist Zurich between July 2010 and July 2015. DCM patients were recruited at $>1$ year after onset of symptoms and tSCI patients were recruited at least 2 months after injury (Table 1). All tSCI patients underwent decompressive surgery before study enrollment while all DCM patients were in pre-operative phase.

The exclusion criteria for both groups were pregnancy, head or brain lesions associated with spinal cord injury, pre-existing neurological and medical disorders leading to functional impairments, mental disorder, or contraindications to MRI, and age $<18$ and $>70$ years.

The tSCI patients were divided in two subgroups based on the severity of impairment: AIS A\&B group (i.e., motor complete) including 10 tSCI patients and AIS C\&D group (i.e., motor incomplete) including $19 \mathrm{tSCI}$ patients to better account for the severity of tSCI. A subset of subjects (17 tSCI patients and $20 \mathrm{DCM}$ patients) included in the present study have been previously presented showing cord tissue specific changes induced by spinal cord injury in $\mathrm{tSCI}^{23}$ or cord myelopathy in DCM. ${ }^{27}$ Additionally, 22 healthy controls (mean age $41.1 \pm 11.4$ years, eight female) were enrolled to confirm the group difference between patients and healthy controls. ${ }^{23,27}$

All patients underwent comprehensive clinical protocols to assess neurologic and functional impairment. These included the
International Standards for Neurological Classification of Spinal Cord Injury (ISNCSCI) protocol for motor score, light-touch, and pinprick score and completeness of injury; GRASSP (maximum 232 points) as ancillary outcome measures dedicated for the assessment of upper limb function ${ }^{19}$ and the Spinal Cord Independence Measure (SCIM). Additionally, all DCM patients were assessed using the modified Japanese Orthopedic Association (mJOA) scale (maximum 18 points). The outcome measures such as the ISNCSCI protocol for the upper extremity motor score (UEMS) (e.g. pyramidal dysfunction), light touch, pinprick, SCIM, and the GRASSP protocol were applied in both SCI and DCM patients to enable the comparison between these two etiologies.

The local ethics committee of Zurich, Kantonale Ethikkommission Zürich, approved the study (KEK-ZH-Nr. -2012-0343), and the study protocols were in accordance with the Declaration of Helsinki. Informed written consent was obtained from each subject before participation.

\section{MRI measurements}

Participants were positioned head-first supine and acquisitions were conducted on a 3T MRI system (SkyraFit Siemens Healthcare, Erlangen, Germany). Radio Frequency (RF) excitation was performed using the body coil and detection was achieved using a combination of 12-channel head-coil, four-channel neck-coil, and 24-channel spine matrix. Subjects were stabilized with an MRIcompatible stifneck (Laerdal Medicals, Stavanger, Norway) to minimize motion artefact effects. As a result of motion artefacts, four patients (three tSCI patients and one DCM patients) and one control were excluded from microstructural assessment.

Table 1. Demographic and Clinical Information of the Traumatic SCI Patients

\begin{tabular}{|c|c|c|c|c|c|c|c|c|c|c|}
\hline$I D$ & Sex & Age, years & AIS grade & Neurologic injury level & Years since injury & GRASSP & $U E M S$ & $U E L T$ & $U E P P$ & SCIM \\
\hline 1 & Male & 29 & A & $\mathrm{C} 4$ & 1.0 & 21 & 14 & 15 & 13 & 22 \\
\hline 2 & Female & 40 & A & $\mathrm{C} 4$ & 7.0 & 43 & 6 & 26 & 12 & 19 \\
\hline 3 & Male & 25 & A & C7 & 0.8 & 125 & 35 & 28 & 27 & 47 \\
\hline 4 & Male & 34 & A & $\mathrm{C} 4$ & 2.6 & 98 & 22 & 21 & 18 & 30 \\
\hline 5 & Male & 66 & A & C6 & 23.9 & 132 & 38 & 20 & 18 & 62 \\
\hline 6 & Male & 68 & A & C7 & 1.0 & NT & 50 & 28 & 28 & NT \\
\hline 7 & Female & 39 & B & $\mathrm{C} 5$ & 25.0 & 125 & 30 & 28 & 25 & 28 \\
\hline 8 & Male & 50 & $\mathrm{~B}$ & $\mathrm{C} 7$ & 25.1 & 188 & 46 & 30 & 26 & 63 \\
\hline 9 & Male & 53 & B & $\mathrm{C} 5$ & 1.5 & 14 & 11 & 20 & 20 & 0 \\
\hline 10 & Female & 32 & $\mathrm{C}$ & C6 & 1.2 & 92 & 26 & 27 & 24 & 23 \\
\hline 11 & Male & 70 & $\mathrm{C}$ & $\mathrm{C} 2$ & 0.7 & 71 & 20 & 20 & 16 & 19 \\
\hline 12 & Male & 31 & $\mathrm{C}$ & $\mathrm{C} 7$ & 11.3 & NT & 50 & 28 & 26 & 57 \\
\hline 13 & Male & 45 & $\mathrm{C}$ & $\mathrm{C} 4$ & 20.6 & 80 & 22 & 24 & 15 & 27 \\
\hline 14 & Male & 69 & $\mathrm{D}$ & $\mathrm{C} 7$ & 0.2 & 206 & 49 & 26 & 25 & 87 \\
\hline 15 & Male & 60 & $\mathrm{D}$ & C3 & 0.3 & NT & 36 & 20 & 20 & 67 \\
\hline 16 & Female & 63 & D & C6 & 0.3 & 172 & 41 & 32 & 29 & 70 \\
\hline 17 & Male & 67 & $\mathrm{D}$ & $\mathrm{C} 7$ & 12.6 & 183 & 41 & 31 & 32 & 99 \\
\hline 18 & Male & 56 & $\mathrm{D}$ & $\mathrm{C} 2$ & 5.6 & 151 & 38 & 24 & 14 & 40 \\
\hline 19 & Male & 43 & $\mathrm{D}$ & $\mathrm{C} 2$ & 13.1 & 225 & 47 & 23 & 23 & 74 \\
\hline 20 & Male & 27 & $\mathrm{D}$ & $\mathrm{C} 7$ & 4.7 & 189 & 46 & 30 & 32 & 75 \\
\hline 21 & Male & 33 & D & C8 & 3.0 & 232 & 50 & 31 & 32 & 89 \\
\hline 22 & Male & 51 & $\mathrm{D}$ & $\mathrm{C} 1$ & 4.3 & 130 & 39 & 20 & 16 & 100 \\
\hline 23 & Male & 48 & $\mathrm{D}$ & $\mathrm{C} 4$ & 1.8 & 232 & 50 & 32 & 32 & 100 \\
\hline 24 & Male & 50 & $\mathrm{D}$ & $\mathrm{C} 3$ & 7.6 & 136 & 38 & 10 & 10 & 97 \\
\hline 25 & Male & 44 & $\mathrm{D}$ & C6 & 12.2 & NT & 50 & 27 & 28 & 100 \\
\hline 26 & Male & 41 & $\mathrm{D}$ & $\mathrm{C} 8$ & 3.3 & NT & 48 & 18 & 17 & 100 \\
\hline 27 & Male & 52 & $\mathrm{D}$ & C8 & 15.1 & NT & 50 & 28 & 28 & 90 \\
\hline 28 & Male & 43 & D & C6 & 4.6 & NT & 45 & 26 & 25 & 92 \\
\hline 29 & Male & 44 & $\mathrm{D}$ & $\mathrm{C} 4$ & 1.2 & NT & 50 & 28 & 28 & NT \\
\hline
\end{tabular}

SCI, spinal cord injury; American Spinal Injury Association Impairment Scale, GRASSP, Graded Redefined Assessment of Strength, Sensibility and Prehension (maximum, 232 points); UEMS, Upper Extremity Motor Score (maximum, 50 points); UELT, Upper Extremity Light-Touch (maximum, 32 points); SCIM, Spinal Cord Independence Measure (maximum, 100 points). 
All participants underwent a protocol consisting of a $\mathrm{T} 2 *_{-}$ weighted three-dimensional (3D) multi-echo sequence (multiple echo data image combination; MEDIC) and a diffusion-weighted imaging (DWI) sequence based on the reduced-field of view (FOV) single-shot spin-echo echo planar imaging above the injury and stenosis level. Macrostructural cord neurodegeneration was assessed by determining gray and white matter in the cross-sectional area (SCA) of the cervical cord using T2*-weighted MRI. The T2*weighted images resulted in five high-resolution axial 3D volumes of the cervical cord with a resolution of $0.25 \times 0.25 \times 2.50 \mathrm{~mm}^{3}$ within $2.8 \mathrm{~min}$ acquisition time per volume. MRI parameters were as follows: $\mathrm{FOV}=162 \times 192 \mathrm{~mm}^{2}$, matrix size $=648 \times 768$, repetition time $(\mathrm{TR})=44 \mathrm{msec}$, echo time $(\mathrm{TE})=19 \mathrm{msec}$, flip angle $\alpha=11^{\circ}$, and read-out bandwidth $=260 \mathrm{~Hz}$ per pixel. To quantify microstructural changes of the spinal cord at the identical level, a high-resolution DWI scan was applied with cardiac-gating (based on finger pulse oximetry) resulting in 30 diffusion-weighted images $\left(b=500 \mathrm{sec} / \mathrm{mm}^{2}\right)$ and six b0-weighted images. The DWI sequence parameters were as follows: slice thickness $=5 \mathrm{~mm}$ with $10 \%$ interslice gap, 10 slices perpendicularly oriented to the spine, 5/8 Partial-Fourier Imaging in phase-encoding direction, phase oversampling $=50 \%$, and a cardiac trigger delay $=200 \mathrm{msec}$, acquisition matrix $=176 \times 40, \mathrm{FOV}=133 \times 30 \mathrm{~mm}^{2}$, in-plane resolutions $=$ $0.8 \times 0.8 \mathrm{~mm}^{2}, \mathrm{TE}=73 \mathrm{msec}$, and $\mathrm{TR}=350 \mathrm{msec}$. The triggered DWI data were acquired in blocks of two slices per cardiac cycle. The minimal time between successive triggers was $1800 \mathrm{msec}$. Each DWI dataset was acquired with four averages resulting in 144 images within a nominal total acquisition time of $6.2 \mathrm{~min}$.

\section{Data processing}

Cross-sectional spinal cord area measurement. The serial longitudinal registration in SPM12 (Wellcome Trust Centre for Neuroimaging, University College London, UK) was applied to all $\mathrm{T} 2 *$-weighted images to average the images accounting for intra-participant motion. Jim 6.0 software (Xinapse Systems, Aldwincle, UK) was used to merge the adjacent partitions resulting in 10 contiguous slices (to increase signal to noise ratio [SNR]) and to semi-automatically segment the cross-sectional cervical cord area using an active-surface model after setting a marker in the center of the cord in each of the 10 contiguous slices. ${ }^{28}$ The gray matter and white matter cross-sectional areas were manually segmented. The mean inter-observer and intraobserver reliability for these measures were shown to be in the range of previously reported results (less than 7\%). ${ }^{27,29}$

Diffusion tensor imaging (DTI) measurement. Processing of DWI data was carried out with a modified version of the Matlabbased ACID toolbox within SPM12 optimized for the spinal cord. ${ }^{30}$ First, we reduced the in-plane FOV to $24 \times 24 \mathrm{~mm}^{2}$ to include only spinal cord tissue. Next, diffusion weighted images were slice-wise linearly registered with 3 degrees of freedom-like translation in the frequency- and phase-encoding direction, scaling in the phaseencoding direction to correct for intra-participant motion and eddycurrent artefacts. ${ }^{31}$ A diffusion tensor model was fitted to the DWI data by applying a robust tensor fitting algorithm that accounts for outlier volumes due to motion and physiologic artefacts ${ }^{32}$ and resulted fractional anisotropy (FA), mean diffusivity (MD), axial diffusivity (AD), and radial diffusivity (RD) DTI index maps. The DTI maps were spatially normalized to a self-constructed mean diffusivity template residing in the spinal Montreal Neurological Institute space. ${ }^{33}$ To further refine the accuracy of the registration, a manual slice-by-slice registration (in-plane translation and scaling) was performed. Finally, all DTI index maps were smoothed with a full width at half-maximum (FWHM) Gaussian kernel with $0.5 \times 0.5 \times 5 \mathrm{~mm}^{3}$. All images were visually inspected for artefacts, and the analysis was conducted on three slices from each modality at the same level.

\section{Statistical analysis}

Statistical analysis of macrostructural MRI data, demographics and clinical outcome data was performed with Stata 15 (Stata- Corp LP, College Station, TX). The mean age was not statistically different between tSCI and DCM patients (Mann-Whitney U test: $\mathrm{Z}=1.06, p=0.29$ ).

First, we assessed the morphometric differences in cord area, gray matter area, and white matter area between tSCI subgroups and DCM patients by means of analysis of covariance, adjusted for age. For assessing microstructural differences between patient's groups, we used voxel-based analysis of the different DTI indexes (FA, AD, RD) in SPM12, adjusted for age. All statistical parametric maps were initially thresholded with a cluster-defining threshold of $p<0.01$ (uncorrected) and clusters surpassing a cluster threshold of $p<0.05$ (family-wise error corrected) are reported. Next, we used linear regression analysis to investigate the relationship between cord macrostructural and microstructural changes and clinical outcome, adjusted for age. The level of significance was set to $p<0.05$.

Data availability statement. Anonymized grouped data, study protocols, and processing pipelines will be shared by request from a qualified investigator.

\section{Results}

\section{Clinical measures}

Of 29 tSCI patients, seven were complete (AIS A) and 22 incomplete (AIS B-D). The average upper-extremity light-touch (maximum, 32), upper-extremity pin-prick (maximum, 32), and upper-extremity motor scores (maximum, 50$)$ were (mean $\pm \mathrm{SD}$ ) $24.86 \pm 5.36,22.72 \pm 6.66$, and $37.52 \pm 13.21$, respectively. The SCIM (maximum, 100) and total GRASSP (maximum, 232) were $62.1 \pm 31.99$ and $135.48 \pm 66.44$, respectively (Table 1 ).

In DCM patients, the upper-extremity light-touch score (mean $\pm \mathrm{SD}$ ) was $27.70 \pm 4.07$, upper-extremity pin-prick score was $27.30 \pm 3.77$, and upper-extremity motor score was $49.70 \pm 0.57$. The SCIM was $97.85 \pm 4.04$. The total GRASSP score was $220.74 \pm 12.32$ (Table 2). Clinical impairment was additionally assessed based on modified Japanese Orthopedic Association (mJOA) score which identified 10 patients suffering from mild $(\mathrm{mJOA} \geq 15$ [max. 18]), nine from moderate $(\mathrm{mJOA}=12-14)$, and one from severe $(\mathrm{mJOA}<12)$ DCM.

Across group comparison, tSCI patients (divided into AIS A\&B and AIS C\&D) showed worse impairments in upper extremity motor score $(p<0.001$ and $p=0.019$, respectively) and worse GRASSP scores $(p<0.001, p<0.001$, respectively) when compared with DCM patients (Fig. 1). Pin-prick score was lower only in tSCI patients with AIS A\&B compared with DCM patients $(p=0.008)$. In contrast, light-touch score was not significantly different comparing tSCI patients (AIS A\&B and AIS C\&D) and DCM patients ( $p=0.32, p=0.29$, respectively).

\section{Cross-sectional spinal cord area}

We first confirmed findings from previous reports that total cross-sectional spinal cord area, gray matter area, and white matter area are decreased in tSCI patients $(p<0.001)$ and in DCM patients $(p<0.001)$ when compared with the healthy controls. ${ }^{23,27} \mathrm{Be}-$ tween patient groups, the magnitude of remote cord atrophy (i.e., SCA) in tSCI patients (AIS A\&B: $58.9 \pm 11.8 \mathrm{~mm}^{2}$; AIS C\&D: $75.3 \pm 16.7 \mathrm{~mm}^{2}$ ) was not significantly different compared with DCM patients (DCM: $68.2 \pm 10.4 \mathrm{~mm}^{2}$; AIS A\&B vs. DCM: $p=0.37$; AIS C\&D vs. DCM: $p=0.40$; Fig. 2 ). 
Table 2. Demographic and Clinical Information of DCM Patients

\begin{tabular}{|c|c|c|c|c|c|c|c|c|c|}
\hline$I D$ & Sex & Age (years) & Stenosis level & GRASSP & $m J O A$ & $U E M S$ & $U E L T$ & $U E P P$ & SCIM \\
\hline 1 & Male & 39 & C3/4* & 225 & 13 & 50 & 25 & 23 & 100 \\
\hline 2 & Female & 53 & $\mathrm{C} 5 / 6$ & 230 & 16 & 50 & 32 & 30 & 100 \\
\hline 3 & Male & 72 & C7/T1* & 222 & 14 & 50 & 26 & 22 & 100 \\
\hline 4 & Female & 37 & C3/4* & 218 & 14 & 49 & 30 & 30 & 99 \\
\hline 5 & Female & 58 & $\mathrm{C} 5 / 6^{*}$ & 220 & 16 & 49 & 29 & 29 & 100 \\
\hline 6 & Male & 55 & C6/7 & 187 & 12 & 50 & 23 & 23 & 98 \\
\hline 7 & Female & 47 & $\mathrm{C} 5 / 6^{*}$ & 232 & 16 & 50 & 31 & 31 & 100 \\
\hline 8 & Male & 63 & $\mathrm{C} 4 / 5^{*}$ & 219 & 12 & 50 & 24 & 24 & 95 \\
\hline 9 & Male & 74 & C6/7* & 215 & 14 & 50 & 32 & 32 & 88 \\
\hline 10 & Male & 32 & $\mathrm{C} 5 / 6^{*}$ & 232 & 16 & 50 & 20 & 20 & 100 \\
\hline 11 & Male & 66 & $\mathrm{C} 5 / 6$ & 215 & 9 & 50 & 25 & 26 & 86 \\
\hline 12 & Male & 36 & C5/6* & 195 & 12 & 48 & 20 & 25 & 99 \\
\hline 13 & Male & 50 & $\mathrm{C} 5 / 6^{*}$ & 231 & 16 & 49 & 25 & 26 & 100 \\
\hline 14 & Male & 51 & C5/6 & 217 & 15 & 50 & 27 & 27 & 96 \\
\hline 15 & Female & 66 & C5/6 & 216 & 12 & 49 & 32 & 32 & 96 \\
\hline 16 & Male & 69 & C5/6* & 227 & 17 & 50 & 32 & 32 & 100 \\
\hline 17 & Male & 68 & C6/7* & NT & 17 & 50 & 32 & 32 & 100 \\
\hline 18 & Male & 39 & C5/6 & 230 & 16 & 50 & 32 & 25 & 100 \\
\hline 19 & Male & 34 & $\mathrm{C} 5 / 6$ & 231 & 14 & 50 & 30 & 30 & 100 \\
\hline 20 & Female & 31 & $\mathrm{C} 5 / 6$ & 232 & 16 & 50 & 27 & 27 & 100 \\
\hline
\end{tabular}

*Multi-segmental degeneration of cervical spine.

GRASSP, Graded Redefined Assessment of Strength, Sensibility and Prehension (maximum, 232 points); mJOA, modified Japanese Orthopedic Association (maximum, 18 points); UEMS, Upper Extremity Motor Score (maximum, 50 points); UELT, Upper Extremity Light-Touch (maximum, 32 points); UEPP, Upper Extremity Pin-Prick (maximum, 32 points); SCIM, Spinal Cord Independence Measure (maximum, 100 points).

Accordingly, the difference between the magnitude of atrophy in gray matter area (AIS A\&B: $10.6 \pm 2.6 \mathrm{~mm}^{2}$, AIS C\&D: $12.8 \pm$ $1.8 \mathrm{~mm}^{2}$, DCM: $12.4 \pm 1.6 \mathrm{~mm}^{2}$; AIS A\&B vs. DCM: $p=0.07$; AIS C\&D vs. DCM: $p=0.95$, respectively) and in white matter area (AIS A\&B: $48.3 \pm 10.2 \mathrm{~mm}^{2}$, AIS C\&D: $63.3 \pm 15.3 \mathrm{~mm}^{2}$, DCM: $55,8 \pm 9.2 \mathrm{~mm}^{2}$, AIS A\&B vs. DCM: $p=0.57$; AIS C\&D vs. DCM: $p=0.22$, respectively) were not significantly different when comparing tSCI to DCM patients.

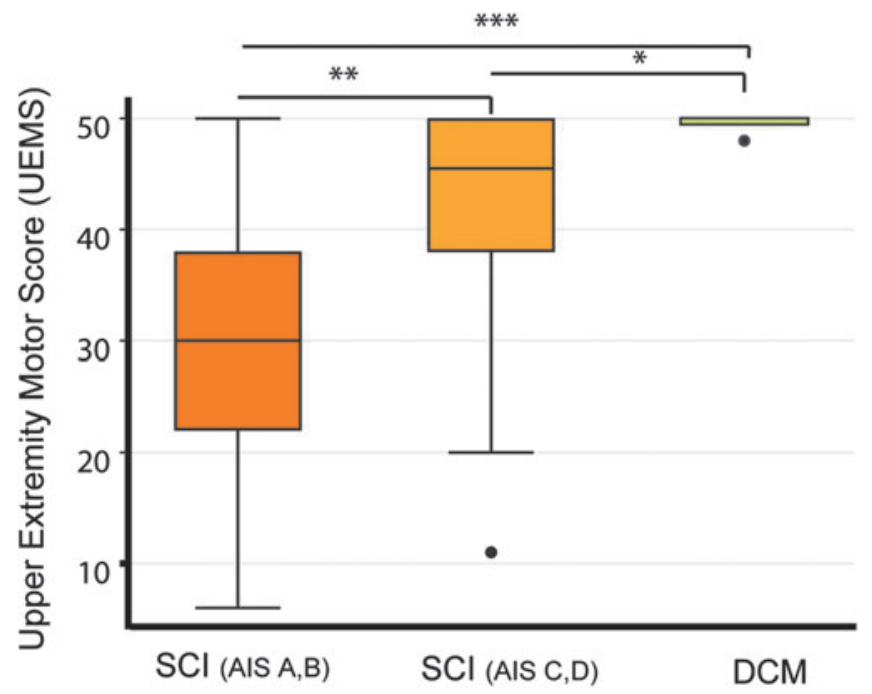

\section{Microstructural neurodegeneration}

We first confirmed by means of voxel-based analysis of the cervical cord DTI data that tSCI and DCM patients show microstructural neurodegenerative changes when compared with healthy controls. $^{23,27}$ Specifically, we found that tSCI patients had a $16 \%$ decrease in FA $(p<0.0001$; localization: $\mathrm{x}=6.4, \mathrm{y}=-19.6, \mathrm{z}=37$; $\mathrm{Z}$-score $=4.70$; cluster extent $=456)$ and a $14 \%$ decrease in $\mathrm{AD}$

FIG. 1. Box plots of UEMS and total GRASSP scores in spinal cord injury (SCI) and degenerative cervical myelopathy (DCM) patients. (A) UEMS is significantly lower in both SCI groups (AIS A\&B and AIS C\&D) compared with DCM patients. Additionally, UEMS of SCI with AIS A\&B shows significant difference compared with SCI with AIS C\&D. (B) GRASSP in SCI (AIS A\&B and C\&D groups) shows significant difference compared with the DCM patients, and there is a difference between the two SCI groups as well. UEMS, Upper Extremity Motor Scores; GRASSP, Graded Redefined Assessment of Strength, Sensation and Prehension; AIS, American Spinal Injury Association Impairment Scale. ${ }^{*} p<0.01 ; * *<0.01$; $* * *<0.001$. Color image is available online. 
A

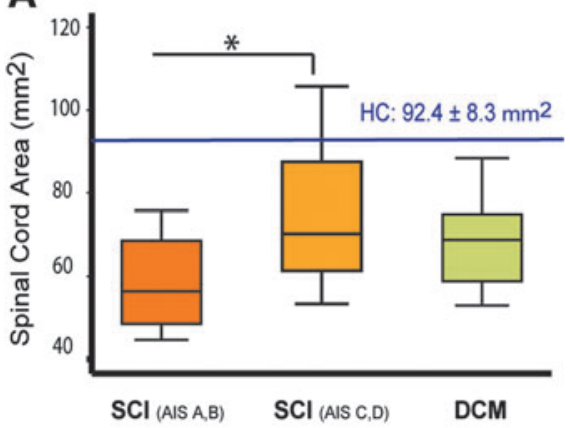

B

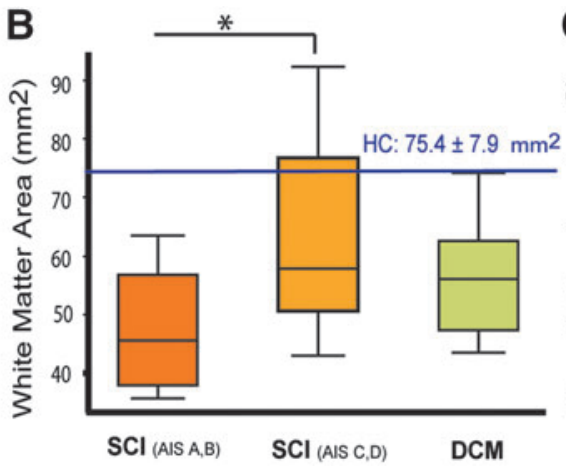

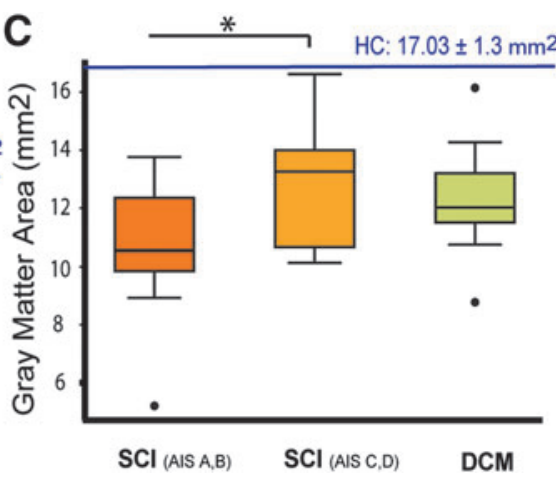

FIG. 2. Box plots of averaged cross-sectional spinal cord, gray matter, and white matter area in spinal cord injury (SCI) and DCM patients (A-C) Smaller spinal cord, gray matter, and white matter area is observed in severely impaired SCI with AIS A\&B grade compared with those in SCI with AIS C\&D grade. However, there is no significant difference in cord atrophy comparing SCI and DCM groups. HC, healthy controls; AIS, American Spinal Injury Association Impairment Scale; DCM, degenerative cervical myelopathy. $(* p<0.01 ; * * p<0.01 ; * * * p<0.001$. Color image is available online.

$(p=0.001 ;$ localization: $\mathrm{x}=4.5, \mathrm{y}=-20.0, \mathrm{z}=15 ; \mathrm{Z}$-score $=4.77$; cluster extent $=234$ ) in the dorsal columns, lateral spinothalamic, and corticospinal tract (CST) tracts when compared with healthy controls. In DCM patients, FA decreased by $18 \%$ in the lateral $\mathrm{CST}$ and spinothalamic tract when compared with healthy controls $(p=0.023$; localization: $\mathrm{x}=4.1, \mathrm{y}=-17.0, \mathrm{z}=21$; Z-score $=$ 3.43; cluster extent $=105$ ).

Comparing tSCI with DCM patients, we found that in tSCI patients, $\mathrm{AD}$ was lower in the dorsal columns (AIS A\&B=-14.4\%; $p=0.005$, localization: $\mathrm{x}=-0.1, \mathrm{y}=-21.9, \mathrm{z}=15$; Z-score $=3.39$; cluster extent $=157$ ) and AIS C\&D $=-12.6 \%, p<0.001$, and in the lateral corticospinal tract (AIS C\&D $=-11.1 \%, p=0.041$; localization: $\mathrm{x}=1.5, \mathrm{y}=-22.6, \mathrm{z}=26 ; \mathrm{Z}$-score $=4.33$; cluster extent $=467$ ). FA in dorsal column was lower only in severely impaired tSCI patients compared with DCM patients (AIS A\&B group $=-18.1 \%, p=0.001$; localization: $\mathrm{x}=0.7, \mathrm{y}=-21.9, \mathrm{z}=21$; $Z$-score $=3.84$; cluster extent $=201$; Fig. 3 ). There were no significant differences between RD measured in $\mathrm{TSCI}$ and DCM patients.

\section{Relationship between remote neurodegeneration and clinical outcomes}

Across all patients (tSCI and DCM), cervical cord gray matter atrophy was associated with upper extremity motor score $(p=0.016$,
$\mathrm{R}^{2}=0.2 ; 95 \%$ confidence interval [CI]: 0.38-3.58, adjusted for age) and GRASSP score $\left(p=0.034, \mathrm{R}^{2}=0.12 ; 95 \%\right.$ CI: $0.36-8.61$, adjusted for age; Fig. 4). Mean FA within corticospinal tracts and dorsal columns was associated with upper extremity motor score $\left(p=0.008, \mathrm{R}^{2}=0.21 ; 95 \%\right.$ CI. 7.38-79.30) and SCIM score $\left(p=0.002, \mathrm{R}^{2}=0.27 ; 95 \% \mathrm{CI}: 66.57-232.47\right.$, adjusted for age $)$ Microstructural and macrostructural changes in DCM patients were not significantly correlated with corresponding clinical impairments.

\section{Discussion}

This study shows extensive cord pathology above a traumatic and non-traumatic cervical spinal cord injury. While macrostructural MRI measures revealed a similar extent of remote cord atrophy, microstructural qMRI measures were able to distinguish more pronounced tract-specific neurodegeneration in tSCI patients. The discrepancy between different clinical presentation and extensive cord pathology in tSCI and DCM patients may be suggestive of compensatory mechanisms owing to the slowly progressing disease in DCM, in contrast to the blunt and abrupt neuronal damage in tSCI patients. Our findings suggest that measures of cord atrophy are insensitive to reveal disease-specific changes while advanced qMRI measures are sensitive to the underlying disease process, as it can detect tract-specific changes.

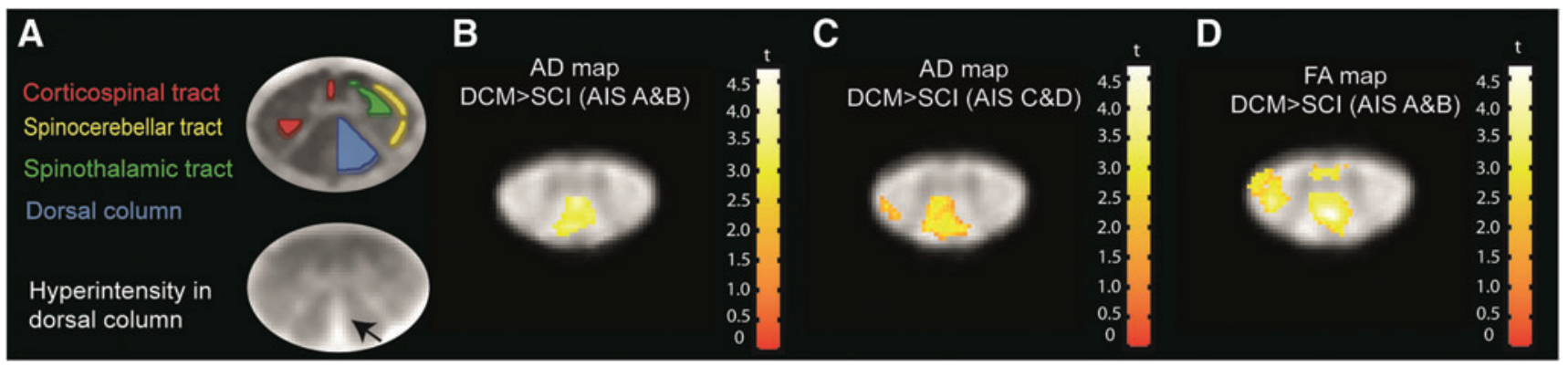

FIG. 3. Voxel-wise analysis of microstructural changes above the level of injury (C2/C3 level) overlaid on the averaged fractional anisotropy maps across subjects in traumatic spinal cord injury (SCI) patients compared with degenerative cervical myelopathy (DCM) patients. (A) white matter atlas and hyperintensity signal on the T2*-weighted scan of a tSCI patient; (B and C) Decreased axial diffusivity (AD) in both SCI groups (American Spinal Injury Association Impairment Scale [AIS] A\&B, $p=0.005 ;$ AIS C\&D, $p<0.001$ ) in dorsal columns and corticospinal tract compared with DCM patients. (D) Decreased fractional anisotropy (FA) in SCI group with AIS A\&B grade compared with DCM patients in dorsal columns $(p<0.001)$. For illustration purpose, the displayed t values are uncorrected at the threshold of $p=0.01$. Color image is available online. 
A

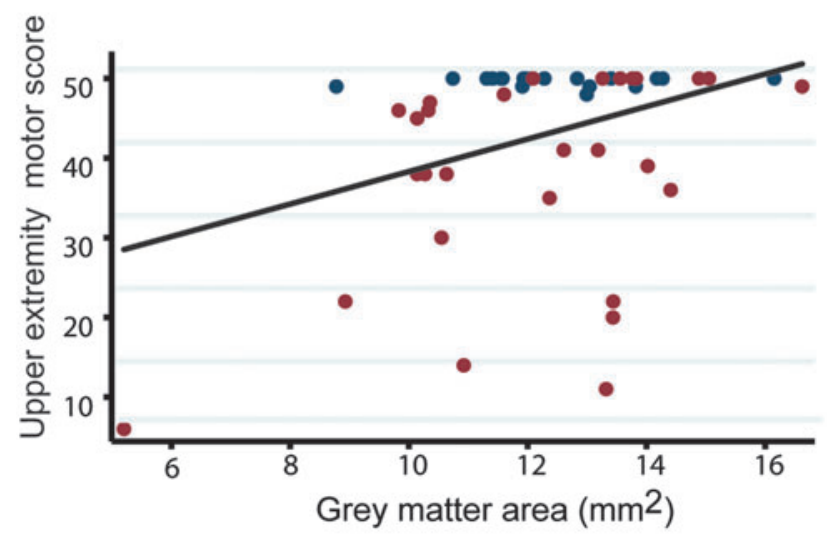

C

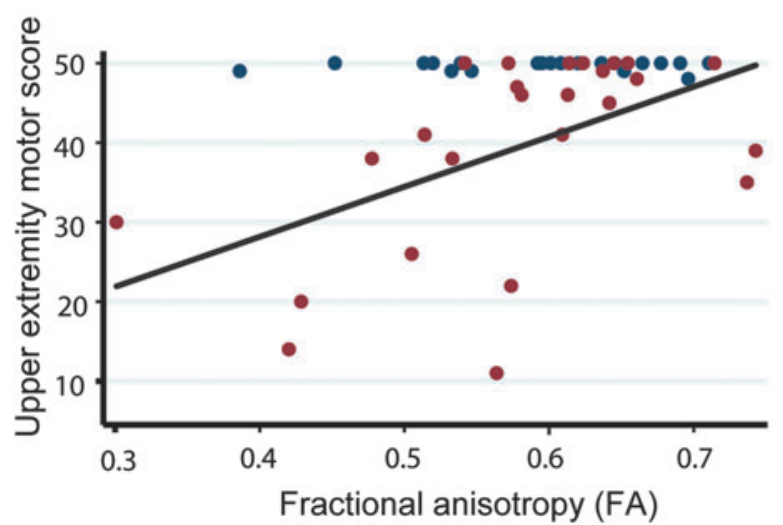

B
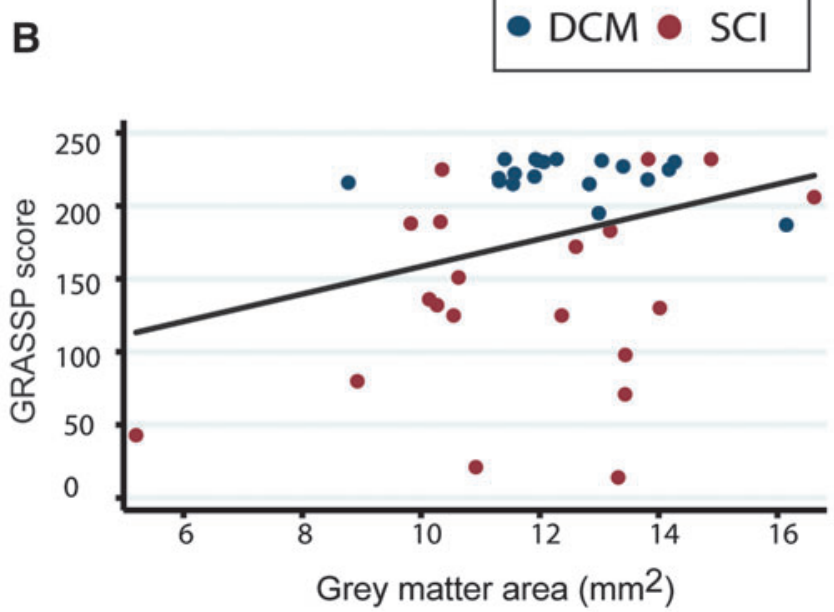

D

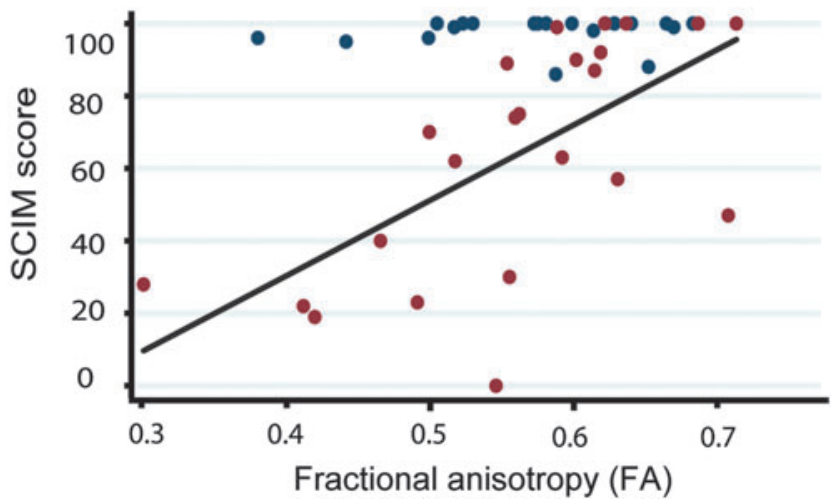

FIG. 4. Associations between remote macrostructural and microstructural magnetic resonance imaging parameters above the level of injury (C2/C3) and clinical impairments in spinal cord injury (SCI) and degenerative cervical myelopathy patients. (A and B) the correlation between gray matter area and upper extremity motor scores (UEMS; $p=0.016, \mathrm{R}^{2}=0.2$ ); and total Graded Redefined Assessment of Strength, Sensibility and Prehension (GRASSP) score $\left(p=0.034, \mathbf{R}^{2}=0.12\right)$. (C and $\left.\mathbf{D}\right)$ Correlation between fractional anisotropy (FA) derived from the corticospinal tract and dorsal columns and UEMS $\left(p=0.008, \mathrm{R}^{2}=0.21\right)$ and total SCIM scores $\left(p=0.002, \mathbf{R}^{2}=0.27\right)$, respectively. Color image is available online.

Cervical cord atrophy remote from the injury site was previously reported in $\mathrm{tSCI}^{21,23}$ and DCM patients ${ }^{27,34}$ when compared with the healthy controls. Here, we also confirmed that the spinal cord atrophy above a cervical injury is different in both patient groups when compared with the healthy controls. However, the remote cord atrophy above the injury level is remarkably similar when tSCI patients are compared with mild DCM patients.

At the microstructural level, previous studies showed that neurodegenerative changes in remote cord regions are evident in $\mathrm{tSCI}^{23,35}$ and in DCM patients. ${ }^{25,27,34}$ In this study we show that albeit similar macrostructural cord changes, the microstructural integrity of the cord is more disturbed in tSCI when compared with DCM above the level of injury. In particular, measures of AD (indicating axonal degeneration) in the dorsal column and lateral corticospinal tract were reduced in tSCI compared with DCM. In addition, FA (indicating axonal count and myelin content) ${ }^{36}$ in dorsal column was significantly reduced in severe tSCI (AIS A-B) compared with DCM. Measures of AD in the dorsal column and lateral corticospinal tract and $\mathrm{FA}^{36}$ in dorsal column were significantly reduced comparing tSCI with DCM. Previous DTI studies in tSCI and DCM have shown increased RD and decreased FA in the supralesional cervical cord ${ }^{23,27}$ remote from the injury level, whereas $\mathrm{AD}$ values changed differently in tSCI patients when compared with DCM. ${ }^{20,24}$ This means that AD remote from the level of stenosis in DCM patients is increased, ${ }^{27}$ while it is decreased in tSCI patients. Increased AD in DCM patients may partially be due to elevated fiber tract density driven by compression and loss of surrounding cord structure, ${ }^{37}$ whereas in tSCI, decreased AD may be due to both axonal loss and demyelination. ${ }^{23,38}$

Despite the differences in etiology, the pathophysiology underlying remote cord atrophy in both tSCI and DCM patients may be driven by similar neurodegenerative mechanisms that are revealed by DTI measures. Pre-clinical studies have highlighted that a range of common primary injury mechanisms are involved in both $\mathrm{tSCI}^{4}$ and DCM patients, ${ }^{15}$ which include apoptosis of cells, inflammation, and vascular changes resulting in cell death at the focal injury site. ${ }^{5,15,39,40}$ Secondary injury-induced changes evolve over time and include anterograde and retrograde axonal degeneration of spinal pathyways, ${ }^{5,14,41,42}$ remodulation of neuronal spinal circuits, ${ }^{43}$ dysregulation of growth factors, ${ }^{39}$ shrinkage of the neuron soma size ${ }^{44}$ due to a reduction in muscle activity of the upper extremity, and remodeling of microvasculature configuration. ${ }^{40}$ 
Interestingly, remote neurodegenerative changes (i.e., atrophy) within the cervical gray matter in both tSCI and DCM groups are associated with upper limb motor function and strength, sensibility, and prehension of the upper limbs (i.e., GRASSP). Microstructural tract-specific changes (FA) above the level of injury also were related to measures of functional independence (i.e., SCIM) and upper limb function. These correlations, though, are mostly driven by SCI patients. Our findings are in line with previous reports showing that MRI derived measures of cord macrostructure and microstructure in the cervical cord are associated with clinical impairments. $^{21,23,27}$ These clinicopathologic associations suggest that remote reorganizational changes, such as remodulation of intraspinal circuits, ${ }^{43}$ contribute to the level of upper limb function in traumatic and non-traumatic SCI. Demonstrating a link between microstructure and function by means of DTI and advanced clinical measures of upper limb function (e.g., GRASSP) points towards the applicability of such advanced qMRI measures over conventional MRI methods in clinical routines. Thus, spinal cord DTI can complement conventional MRI, with the potential to enhance current diagnosis and more importantly, predict outcome in tSCI and DCM patients. In particular, FA was found to show the strongest correlation with clinical scores (ISNCSCI scores in tSCI, mJOA and Nurick scores in DCM), where lower FA values were associated with higher impairment. Neuroimaging biomarkers sensitive to sensorimotor functions could therefore be used for the prediction of upper limb recovery and stratification for interventional trials.

This study has some limitations. DCM patients were on average 5 years older than tSCI patients. Therefore, age was considered as a covariate of no interest in all statistical analyses. Consequently, voxel-based analysis of DTI indexes in the spinal cord are still under development for the spatial normalization of the spinal cord images into a common space; an automated post-processing pipeline is a work in progress. To increase the reliability of our analysis, we therefore manually corrected the spatial normalization to the template.

\section{Conclusion}

Despite clinical differences in traumatic and non-traumatic SCI patients, cord atrophy rostral to the level of the cervical injury is similar. However, measures of cord atrophy represent an accumulation of pathophysiological changes, and as such are insensitive to reveal disease specific changes. On the contrary, advanced qMRI measures can detect tract-specific changes that are clinically eloquent. Thus, DTI of the cervical cord might be a suitable biomarker for outcome prediction and to monitor treatment effects in interventional trials in both traumatic and non-traumatic SCI.

\section{Acknowledgments}

We would like to thank all participants in our study who gave generously of their time, the staff of the Department of Radiology as well as Dr. Markus Hupp and Dr. Katharina Wolf at the Spinal Cord Injury Center Balgrist for their help in patient recruitment.

\section{Funding Information}

This study is funded by ERA-NET NEURON (hMRIofSCI no: 32NE30_173678), the European Union's Horizon 2020 research and innovation program under the grant agreement No 681094, and the Swiss State Secretariat for Education, Research and Innovation (SERI) under contract number 15.0137, grants from Wings for life charity (INSPIRED) (No WFL-CH-007/14), grants from International Foundation for Research (IRP-158). Patrick Freund is sponsored by the Eccellenza fellowship/181362 by SNSF. Open access for this article was funded by Wellcome Trust, grant 091593/Z/10/Z

\section{Author Disclosure Statement}

No competing financial interests exist.

\section{References}

1. Fawcett, J.W., Curt, A., Steeves, J.D., Coleman, W.P., Tuszynski, M.H., Lammertse, D., Bartlett, P.F., Blight, A.R., Dietz, V., Ditunno, J., Dobkin, B.H., Havton, L.A., Ellaway, P.H., Fehlings, M.G., Privat, A., Grossman, R., Guest, J.D., Kleitman, N., Nakamura, M., Gaviria, M., and Short, D. (2007). Guidelines for the conduct of clinical trials for spinal cord injury as developed by the ICCP panel: spontaneous recovery after spinal cord injury and statistical power needed for therapeutic clinical trials. Spinal Cord 45, 190-205.

2. Kato, S. and Fehlings, M. (2016). Degenerative cervical myelopathy. Curr. Rev. Musculoskelet. Med. 9, 263-271.

3. Ones, K., Yilmaz, E., Beydogan, A., Gultekin, O., and Caglar, N. (2007). Comparison of functional results in non-traumatic and traumatic spinal cord injury. Disabil. Rehabil. 29, 1185-1191.

4. Ahuja, C.S., Wilson, J.R., Nori, S., Kotter, M.R.N., Druschel, C., Curt, A., and Fehlings, M.G. (2017). Traumatic spinal cord injury. Nat. Rev. Dis. Prim. 3, 17018.

5. Karadimas, S.K., Moon, E.S., Yu, W.R., Satkunendrarajah, K., Kallitsis, J.K., Gatzounis, G., and Fehlings, M.G. (2013). A novel experimental model of cervical spondylotic myelopathy (CSM) to facilitate translational research. Neurobiol. Dis. 54, 43-58.

6. Talekar, K., Poplawski, M., Hegde, R., Cox, M., and Flanders, A. (2016). Imaging of spinal cord injury: acute cervical spinal cord injury, cervical spondylotic myelopathy, and cord herniation. Semin. Ultrasound CT MRI 37, 431-447.

7. Fehlings, M.G., Martin, A.R., Tetreault, L.A., Aarabi, B., Anderson, P., Arnold, P.M., Brodke, D., Burns, A.S., Chiba, K., Dettori, J.R., Furlan, J.C., Hawryluk, G., Holly, L.T., Howley, S., Jeji, T., KalsiRyan, S., Kotter, M., Kurpad, S., Kwon, B.K., Marino, R.J., Massicotte, E., Merli, G., Middleton, J.W., Nakashima, H., Nagoshi, N., Palmieri, K., Singh, A., Skelly, A.C., Tsai, E.C., Vaccaro, A., Wilson, J.R., Yee, A., and Harrop, J.S. (2017). A clinical practice guideline for the management of patients with acute spinal cord injury: recommendations on the role of baseline magnetic resonance imaging in clinical decision making and outcome prediction. Glob. Spine J. 7, 221S-230S.

8. Kalsi-Ryan, S., Karadimas, S.K., and Fehlings, M.G. (2013). Cervical spondylotic myelopathy: the clinical phenomenon and the current pathobiology of an increasingly prevalent and devastating disorder. Neuroscientist 19, 409-421.

9. Lemon, R.N. and Griffiths, J. (2005). Comparing the function of the corticospinal system in different species: organizational differences for motor specialization? Muscle Nerve 32, 261-79.

10. Starkey, M.L. and Schwab, M.E. (2012). Anti-Nogo-A and training: can one plus one equal three? Exp. Neurol. 235, 53-61.

11. Karadimas, S.K., Gatzounis, G., and Fehlings, M.G. (2015). Pathobiology of cervical spondylotic myelopathy. Eur. Spine J. 24, 132138.

12. Yu, W.R., Liu, T., Kiehl, T.R., and Fehlings, M.G. (2011). Human neuropathological and animal model evidence supporting a role for Fas-mediated apoptosis and inflammation in cervical spondylotic myelopathy. Brain 134, 1277-1292.

13. Bohlman, H.H. (1988). The pathophysiology of cervical spondylosis and myelopathy. Spine (Phila. Pa. 1976). 13, 726-730.

14. Fehlings, M.G. and Skaf, G. (1998). A review of the pathophysiology of cervical spondylotic myelopathy with insights for potential novel mechanisms drawn from traumatic spinal cord injury. Spine (Phila. Pa. 1976). 23, 2730-2737.

15. Akter, F. and Kotter, M. (2018). Pathobiology of Degenerative Cervical Myelopathy. Neurosurg. Clin. N. Am. 29, 13-19.

16. Kalsi-Ryan, S., Singh, A., Massicotte, E.M., Arnold, P.M., Brodke, D.S., Norvell, D.C., Hermsmeyer, J.T., and Fehlings, M.G. (2013). Ancillary outcome measures for assessment of individuals with cer- 
vical spondylotic myelopathy. Spine (Phila. Pa. 1976). 38, S111S122.

17. Petersen, J.A., Wilm, B.J., von Meyenburg, J., Schubert, M., Seifert, B., Najafi, Y., Dietz, V., and Kollias, S. (2012). Chronic cervical spinal cord injury: DTI correlates with clinical and electrophysiological measures. J. Neurotrauma 29, 1556-1566.

18. Curt, A., Keck, M.E., and Dietz, V. (1998). Functional outcome following spinal cord injury: significance of motor-evoked potentials and ASIA scores. Arch. Phys. Med. Rehabil. 79, 81-86.

19. Kalsi-Ryan, S., Curt, A., Verrier, M.C., and Fehlings, M.G. (2012). Development of the Graded Redefined Assessment of Strength, Sensibility and Prehension (GRASSP): reviewing measurement specific to the upper limb in tetraplegia. J. Neurosurg. Spine 17, 65-76.

20. Kalsi-Ryan, S., Curt, A., Fehlings, M., and Verrier, M. (2009). Assessment of the hand in tetraplegia using the graded redefined assessment of strength, sensibility and prehension (GRASSP). Top. Spinal Cord Inj. Rehabil. 14, 34-46.

21. Freund, P., Weiskopf, N., Ashburner, J., Wolf, K., Sutter, R., Altmann, D.R., Friston, K., Thompson, A., Curt, A., Freund, P., Weiskopf, N., Ashburner, J., and Friston, K. (2013). MRI investigation of the sensorimotor cortex and the corticospinal tract after acute spinal cord injury: a prospective longitudinal study. Lancet Neurol. 12, 873-881.

22. Seif, M., Leutritz, T., Samson, R.S., Wheeler-Kingshott, C.A.M., Curt, A., Freund, P., and Weiskopf, N. (2018). A multi-center study on fast full-brain quantitative multi-parameter mapping of R1, MT, and R2*: scan-rescan repeatability and inter-site reproducibility, in: Proceedings of the 2018 Annual Meeting of the International Society for Magnetic Resonance in Medicine, pp. 1119.

23. Huber, E., David, G., Thompson, A.J., Weiskopf, N., Mohammadi, S., and Freund, P. (2018). Dorsal and ventral horn atrophy is associated with clinical outcome after spinal cord injury. Neurology 90, e1510 e1522.

24. Cohen-Adad, J. (2018). Microstructural imaging in the spinal cord and validation strategies. Neuroimage 182, 169-183.

25. Martin, A.R., De Leener, B., Cohen-Adad, J., Cadotte, D.W., Nouri, A., Wilson, J.R., Tetreault, L., Crawley, A.P., Mikulis, D.J., Ginsberg, H., and Fehlings, M.G. (2018). Can microstructural MRI detect subclinical tissue injury in subjects with asymptomatic cervical spinal cord compression? A prospective cohort study. BMJ Open 8, e019809.

26. Martin, A.R., De Leener, B., Cohen-Adad, J., Cadotte, D.W., KalsiRyan, S., Lange, S.F., Tetreault, L., Nouri, A., Crawley, A., Mikulis, D.J., Ginsberg, H., and Fehlings, M.G. (2017). Clinically feasible microstructural MRI to quantify cervical spinal cord tissue injury using DTI, MT, and T2*-weighted imaging: assessment of normative data and reliability. Am. J. Neuroradiol. 38, 1257-1265.

27. Grabher, P., Mohammadi, S., Trachsler, A., Friedl, S., David, G., Sutter, R., Weiskopf, N., Thompson, A.J., Curt, A., and Freund, P. (2016). Voxel-based analysis of grey and white matter degeneration in cervical spondylotic myelopathy. Sci. Rep. 6, 24636.

28. Horsfield, M.A., Sala, S., Neema, M., Absinta, M., Bakshi, A., Sormani, M.P., Rocca, M.A., Bakshi, R., and Filippi, M. (2010). Rapid semi-automatic segmentation of the spinal cord from magnetic resonance images: Application in multiple sclerosis. Neuroimage 50, 446455.

29. Yiannakas, M.C., Kearney, H., Samson, R.S., Chard, D.T., Ciccarelli, O., Miller, D.H., and Wheeler-Kingshott, C.A.M. (2012). Feasibility of grey matter and white matter segmentation of the upper cervical cord in vivo: a pilot study with application to magnetisation transfer measurements. Neuroimage 63, 1054-1059.

30. David, G., Freund, P., and Mohammadi, S. (2017). The efficiency of retrospective artifact correction methods in improving the statistical power of between-group differences in spinal cord DTI. Neuroimage 158, 296-307.

31. Mohammadi, S., Möller, H.E., Kugel, H., Müller, D.K., and Deppe, M. (2010). Correcting eddy current and motion effects by affine whole-brain registrations: Evaluation of three-dimensional distortions and comparison with slicewise correction. Magn. Reson. Med. 64, 1047-1056.

32. Kamble, R.B., Venkataramana, N.K., Naik, A.L., and Rao, S. V. (2011). Diffusion tensor imaging in spinal cord injury. Indian J. Radiol. Imaging 21, 221-224.

33. Fonov, V.S., Le Troter, A., Taso, M., De Leener, B., Lévêque, G., Benhamou, M., Sdika, M., Benali, H., Pradat, P.F., Collins, D.L., Callot, V., and Cohen-Adad, J. (2014). Framework for integrated MRI average of the spinal cord white and gray matter: the MNI-Poly-AMU template. Neuroimage 102, 817-827.

34. Martin, A., Leener, B. De, Cohen-Adad, J., Kalsi-Ryan, S., Cadotte, D.W., Wilson, J.R., Tetreault, L.A., Nouri, A., Crawley, A., Mikulis, D.J., Ginsberg, H., Massicotte, E.M., and Fehlings, M.G. (2018). Monitoring for myelopathic progression with multiparametric quantitative MRI. PLoS One 3, e0195733

35. Cohen-Adad, J., El Mendili, M.M., Lehéricy, S., Pradat, P.F., Blancho, S., Rossignol, S., and Benali, H. (2011). Demyelination and degeneration in the injured human spinal cord detected with diffusion and magnetization transfer MRI. Neuroimage 55, 1024-1033.

36. Schmierer, K., Wheeler-Kingshott, C.A.M., Boulby, P.A., Scaravilli, F., Altmann, D.R., Barker, G.J., Tofts, P.S., and Miller, D.H. (2007). Diffusion tensor imaging of post mortem multiple sclerosis brain. Neuroimage 35, 467-477.

37. Ellingson, B.M., Salamon, N., Woodworth, D.C., and Holly, L.T. (2015). Correlation between degree of subvoxel spinal cord compression measured with super-resolution tract density imaging and neurological impairment in cervical spondylotic myelopathy. J. Neurosurg. Spine 22, 631-638.

38. Brennan, F.H., Cowin, G.J., Kurniawan, N.D., and Ruitenberg, M.J. (2013). Longitudinal assessment of white matter pathology in the injured mouse spinal cord through ultra-high field (16.4T) in vivo diffusion tensor imaging. Neuroimage 82, 574-585.

39. Bareyre, F.M. and Schwab, M.E. (2003). Inflammation, degeneration and regeneration in the injured spinal cord: insights from DNA microarrays. Trends Neurosci. 26, 555-563.

40. Cao, Y., Zhou, Y., Ni, S., Wu, T., Li, P., Liao, S., Hu, J., and Lu, H. (2017). Three dimensional quantification of microarchitecture and vessel regeneration by synchrotron radiation microcomputed tomography in a rat model of spinal cord injury. J. Neurotrauma 34, 1187-1199.

41. Bunge, R.P., Puckett, W.R., Becerra, J.L., Marcillo, A., and Quencer, R.M. (1993). Observations on the pathology of human spinal cord injury. A review and classification of 22 new cases with details from a case of chronic cord compression with extensive focal demyelination. Adv. Neurol. 59, 75-89.

42. Buss, A., Pech, K., Merkler, D., Kakulas, B.A., Martin, D., Schoenen, J., Noth, J., Schwab, M.E., and Brook, G.A. (2005). Sequential loss of myelin proteins during Wallerian degeneration in the human spinal cord. Brain 128, 356-364.

43. Bareyre, F.M., Kerschensteiner, M., Raineteau, O., Mettenleiter, T.C., Weinmann, O., and Schwab, M.E. (2004). The injured spinal cord spontaneously forms a new intraspinal circuit in adult rats. Nat. Neurosci. 7, 269-277.

44. Liu, N.-K., Byers, J.S., Lam, T., Lu, Q.B., Sengelaub, D.R., and Xu, X.M. (2014). Inhibition of cPLA2 has neuroprotective effects on motoneuron and muscle atrophy following spinal cord injury. J. Neurotrauma 2014 Nov 11; Epub ahead of print.

Address correspondence to:
Maryam Seif, PhD
Spinal Cord Injury Center
University Hospital Balgrist
Forchstrasse 340
8008 Zurich
Switzerland

E-mail: maryam.seif@balgrist.ch 